\title{
An Inter- and Intra-Subject Transfer Calibration Scheme for Improving Feedback Performance of Sensorimotor Rhythm-Based BCI Rehabilitation
}

\author{
Lei Cao ${ }^{1}$, Shugeng Chen ${ }^{2}$, Jie Jia ${ }^{2 *}$, Chunjiang Fan ${ }^{3 *}$, Haoran Wang ${ }^{4}$ and Zhixiong $\mathrm{Xu}^{1}$ \\ ${ }^{1}$ Department of Artificial Intelligence, Shanghai Maritime University, Shanghai, China, ${ }^{2}$ Department of Rehabilitation Medicine, \\ Huashan Hospital, Fudan University, Shanghai, China, ${ }^{3}$ Wuxi Rehabilitation Hospital, Wuxi, China, ${ }^{4}$ Department of Computer \\ Science and Technology, Tongji University, Shanghai, China
}

\section{OPEN ACCESS}

Edited by:

Yizhang Jiang,

Jiangnan University, China

Reviewed by:

Shangce Gao,

University of Toyama, Japan

Cheng Wang,

Huaqiao University, China

*Correspondence:

Chunjiang Fan

Icao@shmtu.edu.cn

Jie Jia

shannonjj@126.com

Specialty section:

This article was submitted to

Brain Imaging Methods,

a section of the journal

Frontiers in Neuroscience

Received: 15 November 2020 Accepted: 21 December 2020 Published: 28 January 2021

Citation:

Cao L, Chen S, Jia J, Fan C, Wang H and Xu Z (2021) An Inter- and Intra-Subject Transfer Calibration Scheme for Improving Feedback

Performance of Sensorimotor Rhythm-Based BCl Rehabilitation.

Front. Neurosci. 14:629572.

doi: 10.3389/fnins.2020.629572
The Brain Computer Interface $(\mathrm{BCl})$ system is a typical neurophysiological application which helps paralyzed patients with human-machine communication. Stroke patients with motor disabilities are able to perform $\mathrm{BCl}$ tasks for clinical rehabilitation. This paper proposes an effective scheme of transfer calibration for $\mathrm{BCl}$ rehabilitation. The inter- and intra-subject transfer learning approaches can improve the low-precision classification performance for experimental feedback. The results imply that the systematical scheme is positive in increasing the confidence of voluntary training for stroke patients. In addition, it also reduces the time consumption of classifier calibration.

Keywords: $\mathrm{BCl}$, transfer learning, rehabilitation, stroke, classifier calibration

\section{INTRODUCTION}

Brain-computer interface (BCI) is developed as an extrinsic pathway for human-machine interaction in a reliable way (Birbaumer, 2006). It is effective for the disabled to control external devices by neural activities (Buch et al., 2008). Stroke patients with motor disabilities in particular, are able to perform BCI tasks for clinical rehabilitation (Meng et al., 2016). In this treatment, sensorimotor rhythm changes are used as neurological modulation for active intervention (Mane et al., 2019).

During rehabilitation, the patients are requested to attempt or to imagine performing a movement. Then, motor attempt (MA) or motor imagery (MI)-BCI systems will output a synchronized sensory biofeedback (e.g., robotic arm recovery) by a trained classifier based on a prior dataset (Pillette et al., 2020). In the intervention, the functional motor is significantly enabled by neurophysiological activity (Xu et al., 2014). This is an ongoing process of brain plasticity and functional recovery (Remsik et al., 2019). Recent studies have reported the improvement of limb movement for stroke patients using long-term sensorimotor rhythm (SMR)-BCI interventions (Ramos-Murguialday et al., 2013; Pichiorri et al., 2015; Bundy et al., 2017).

Nevertheless, BCI rehabilitation is limited by poor-efficiency recognition algorithms and model-personalized variability (Grosse-Wentrup et al., 2011). Relevant work has proved that BCI decoding accuracy was insufficient for rehabilitation outcomes (Mane et al., 2020). Moreover, the failures of BCI feedback also reduce the confidence of trainees subtly (Foong et al., 2019). Hence, various improvements of pattern recognition and model calibration should be made to enhance SMR-BCI performance in the clinical application. 
Conventionally, SMR features are effectively extracted by a time-frequency analysis for the healthy (Pfurtscheller and Da Silva, 1999). For instance, the event-related desynchronization (ERD) amplitudes oscillated in the $\mu$ rhythm was detected during the motor imagery task for pattern recognition (Huang et al., 2012; Saha et al., 2017). Furthermore, the common spatial pattern (CSP) algorithm was proposed for feature extracting in the spatial domain (Wang et al., 2006; Arvaneh et al., 2013). It was efficient for mining the significant difference of two-type motor tasks. However, the degeneration of neural activation due to post stroke, has a negative impact on BCI performance (Shu et al., 2018). Thus, the signal characteristics are much lower than those of healthy individuals during motor tasks (De Vries et al., 2013; Caria et al., 2020). Therefore, increasing the precision of SMR-BCI using a mathematical methods is meaningful for BCI intervention.

To solve this problem, transfer learning (TL), which applies the dataset in source domains for compensating insufficient labeled data in a target domain, has been proposed for MIBCIs (Samek et al., 2013b; Azab et al., 2018). This technology is developed in several ways, such as instance selection ( $\mathrm{Wu}$, 2016; Hossain et al., 2018), feature calibration (Samek et al., 2013a; Zhao et al., 2019) and classification domains (Vidaurre et al., 2010; He and $\mathrm{Wu}, 2019)$. For instance, for selection, active learning is typically presented for selecting training data from intra- or inter-subject labeled trials (Hossain et al., 2018). The target of this approach is to increase the informative trials of the new subject by adding sufficient existing labeled trials that were close to prior dataset. In the feature domain, transfer calibration approaches mainly concentrate on regulating the covariance matrix estimation and optimization function for improving the performance of CSP models. For example, researchers regularized the CSP filter by the average of the common feature space from other subjects (Kang et al., 2009). Moreover, the efficiency of domain adaptation has been verified for MI-BCI in the classification domain (Vidaurre et al., 2010). Kobler et al. constructed a Restricted Boltzmann Machine (RBM) based on public baseline data and applied it for the MI-BCI task (Kobler and Scherer, 2016). Recently, multi-task learning has been presented in the relevant experiment (Jayaram et al., 2016; Gao et al., 2019), where the weight parameters of intersubjective classifiers were learned jointly for minimizing the dissimilarities between these existing classification models and the target model. However, BCIs controlled by these approaches have been proven to be only valid for healthy individuals. None of them are experimentally evaluated for BCI rehabilitation.

This paper first proposes a transfer calibration scheme to improve the rehabilitation outcomes of SMR-BCI. First, we utilize a transfer learning algorithm, whose effects have been verified in the task of MI-BCI (Azab et al., 2019), to validate the reliability for stroke patients. Then, we discuss the respective applicability between intra- and inter- subjective conditions. The results show that our proposed approaches improved the lowprecision classification performance. Accordingly, we generalize the scheme of transfer calibration for SMR-BCI intervention. This methodology applies for other transfer learning algorithms to increase the precision of BCI feedback.

\section{MATERIALS AND METHODS}

\subsection{Experimental Paradigm and Subjects}

Seven stroke patients aged between 30 and 65, recruited from the Department of Rehabilitation Medicine of Huashan Hospital participated in our experiments (Table 1). All of them were naive to BCI and provided consent to be involved in the study. The inclusion criteria for this study were as follows: (1) unilateral motor dysfunction diagnosed by computer tomography or magnetic resonance imaging (MRI); (2) first onset stroke patient; (3) the time since stroke onset was more than 4 weeks and less than 6 months; (4) the assessment of cognitive functions: MiniMental State Examination score $>25$. The exclusion criteria were listed below: (1) unstable medical conditions; (2) severe vision problems; (3) the intervention treatment by other brain stimulations during the study period.

In the experiment, BCI intervention was performed in three sessions a week for each patient. And it lasted 1 month, with a total of 12 sessions. One session contained three runs, each run had 30 trials for each mental task (motor attempt or idle state) performed in a random order. Subjects underwent two experimental tasks. In the task of motor attempt (MA), patients were required to attempt motion of wrist extension with affected hands continually, but not to have compensatory movements. In the other task of idle state (IS), they needed to do nothing but rest (Figure 1A).

The experimental paradigm is shown in Figure 1B. In one trial, the duration time was about $11 \mathrm{~s}$. The patient was asked to sit in front of a computer screen, with arms resting on a desk. A white arrow presented on the center of the screen from 0 to $3 \mathrm{~s}$. The patient was instructed to keep still and rest. Then, an alternative indicator (a red square or a red rectangle) was displayed in the center of the screen, representing a task (MA or IS). After the command disappeared, the subject was required to perform the corresponding task for $5 \mathrm{~s}$ until the white cross disappeared. Finally, the rest interval was adopted randomly to relax.

\subsection{EEG Recording and Signal Preprocessing}

EEG were collected using 32 channels consisting of $\mathrm{Ag} / \mathrm{AgCl}$ electrode of EEG cap (actiCAP; Brain Products, Germany) according to the configuration of 10-C20 International System. A bio-signal amplifier (Brain Products) was used for acquiring the signals. The unilateral reference electrode was located in the right mastoid process, and the ground electrode was located in the forehead. The other 31 channels (FP1, FZ, F3, F7, FT9, FC5, FC1, C3, T7, TP9, CP5, CP1, PZ, P3, P7, O1, O2, P4, P8, TP10, CP6, CP2, CZ, C4, T8, FT10, FC6, FC2, F4, F8, FP2) were used for calculation. Electrode impedances were kept below $5 \mathrm{k} \Omega$. The signals were amplified, digitalized with a sample rate of $200 \mathrm{~Hz}$, and bandpass-filtered between 1 and $35 \mathrm{~Hz}$.

\subsection{Evaluation of $\mathrm{BCI}$ Performance}

$\mathrm{BCI}$ performance was evaluated by classification accuracy for two mental tasks. As shown in the figure of experimental paradigm, the subject conducted the mental task from $4.5 \mathrm{~s}$ to $9.5 \mathrm{~s}$. Hence, 
TABLE 1 | The baseline clinical characteristics of participants.

\begin{tabular}{|c|c|c|c|c|c|}
\hline Patients & Gender & Affected hand & Type of injury & Time since injury (m) & Site of injury \\
\hline S1 & Male & Right & Ischemia & 5 & Left, basal ganglia \\
\hline S2 & Male & Left & Hemorrhage & 4 & Right, basal ganglia \\
\hline S3 & Male & Right & Hemorrhage & 1 & Left, basal ganglia \\
\hline S4 & Male & Right & Ischemia & 1 & Left, paracele \\
\hline S5 & Male & Right & Ischemia & 3 & Left, basal ganglia \\
\hline S6 & Male & Right & Ischemia & 5 & Left, paracele, basal ganglia \\
\hline S7 & Male & Left & Ischemia & 3 & Right, basal ganglia \\
\hline
\end{tabular}

A

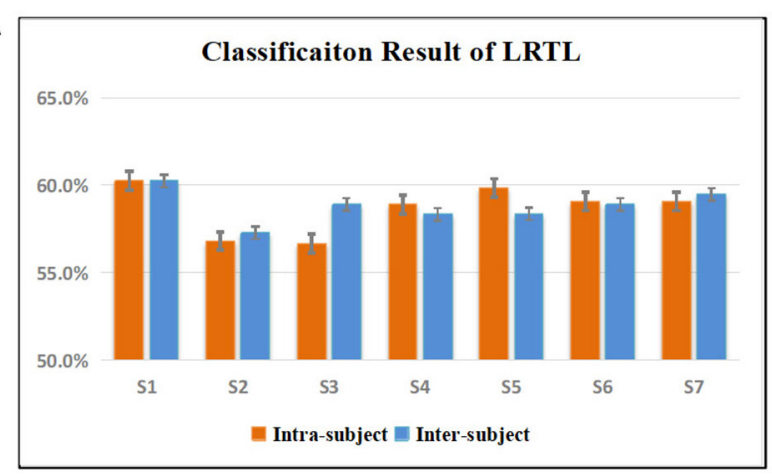

B

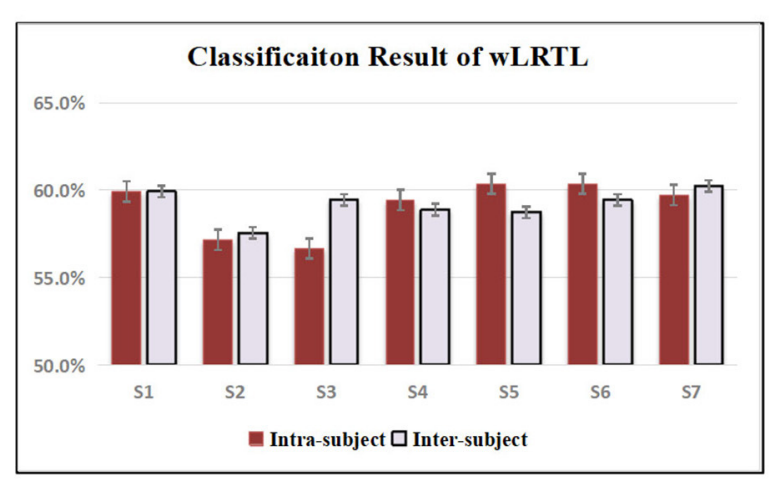

FIGURE 1 | (A) Experimental procedures of two different mental tasks. In the task of MA, patients were required to attempt motion of wrist extension with affected hands continually, but not to have compensatory movements. In the other task of IS, they need not do anything for a rest. (B) Overview of BCl control paradigm.

EEG signals were extracted from [4.5 9.5] s of each single trial. And the features were filtered with the common spatial pattern (CSP) method, whose log-variance of the first and last three components were selected as output vectors. Then, a transfer learning scheme was used to improve the classification efficiency. The Logistic Regression (LR) -based classifier was used as the baseline approach. Its classification parameters $w_{t}$ were calibrated using the following function,

$$
L\left(w_{t}\right)=\min _{w_{t}}\left(\sum_{n_{t}}^{i=1} H\left(w_{t} ; l_{t}^{i}, f_{t}^{i}\right)+\lambda_{t}\left\|w_{t}\right\|_{2}^{2}\right),
$$

where $H, f_{t}, l_{t}$ and $\|.\|_{2}$ denote the cross-entropy, feature vectors extracted from the EEG dataset, label vector, and 2norm functions, respectively. Conventionally, the parameters were supposed be trained by large prior data. Transfer learning algorithms had been proposed for improving the efficiency of parameter calibration of new subjects without subject-specific data (Hossain et al., 2018). In the framework of logistic regression -based transfer learning (LRTL), a regularization term penalizing dissimilarities $R_{t}$ was used for transferring the prior distribution of the existing classification parameters into the calibration of the present training of new target subjects or sessions. In this opinion, the classification parameters were calculated as follows,

$$
L^{\prime}\left(w_{t}\right)=\min _{w_{t}}\left(\sum_{n_{t}}^{i=1} H\left(w_{t} ; l_{i}^{t}, l_{i}^{f}\right)+\lambda_{t} R_{t}\left(w_{t}\right)\right) \text {, }
$$

and the $R_{t}$ was decided by estimating the similarity between feature distribution of existing models and that of current few training data,

$$
R_{t}\left(w_{t}\right)=0.5\left[\left(w_{t}-\mu\right)^{T} \Sigma_{t}^{-1}\left(w_{t}-\mu\right)+\log \left(\left|\Sigma_{t}\right|\right)\right],
$$

where $\mu$ and $\Sigma_{t}$ were, respectively, obtained as

$$
\mu=\frac{1}{n} \sum_{t^{\prime}=1}^{n} w_{t^{\prime}}
$$

$$
\Sigma_{t}=\frac{\operatorname{diag}\left(\Sigma_{t^{\prime}=1}^{n}\left(w_{t^{\prime}}-\mu\right)\left(w_{t^{\prime}}-\mu\right)^{T}\right)}{\operatorname{trace}\left(\Sigma_{t^{\prime}=1}^{n}\left(w_{t^{\prime}}-\mu\right)\left(w_{t^{\prime}}-\mu\right)^{T}\right)} .
$$

Here, $\mathrm{T}$ denoted transpose of the matrix, the functions of diag and trace were defined as the diagonal elements and the sum of the diagonal elements of a matrix. Furthermore, KullbackLeibler (KL) divergence was added to solve the problem of weight distribution between existing models and the target model. It was supposed to give larger weights to more similar distributions and smaller weights to less similar distributions. KL divergence of two EEG sets $\left(E_{0}, E_{1}\right)$ were represented as the following form,

$$
\begin{aligned}
K L\left[E_{0} \| E_{1}\right]= & 0.5\left[\left(\mu_{1}-\mu_{0}\right)^{T} \Sigma_{1}^{-1}\left(\mu_{1}-\mu_{0}\right)+\operatorname{trace}\left(\Sigma_{1}^{-1} \Sigma_{0}\right)\right. \\
& \left.-\ln \left(\frac{\operatorname{det}\left(\Sigma_{0}\right)}{\operatorname{det}\left(\Sigma_{1}\right)}\right)-K\right]
\end{aligned}
$$




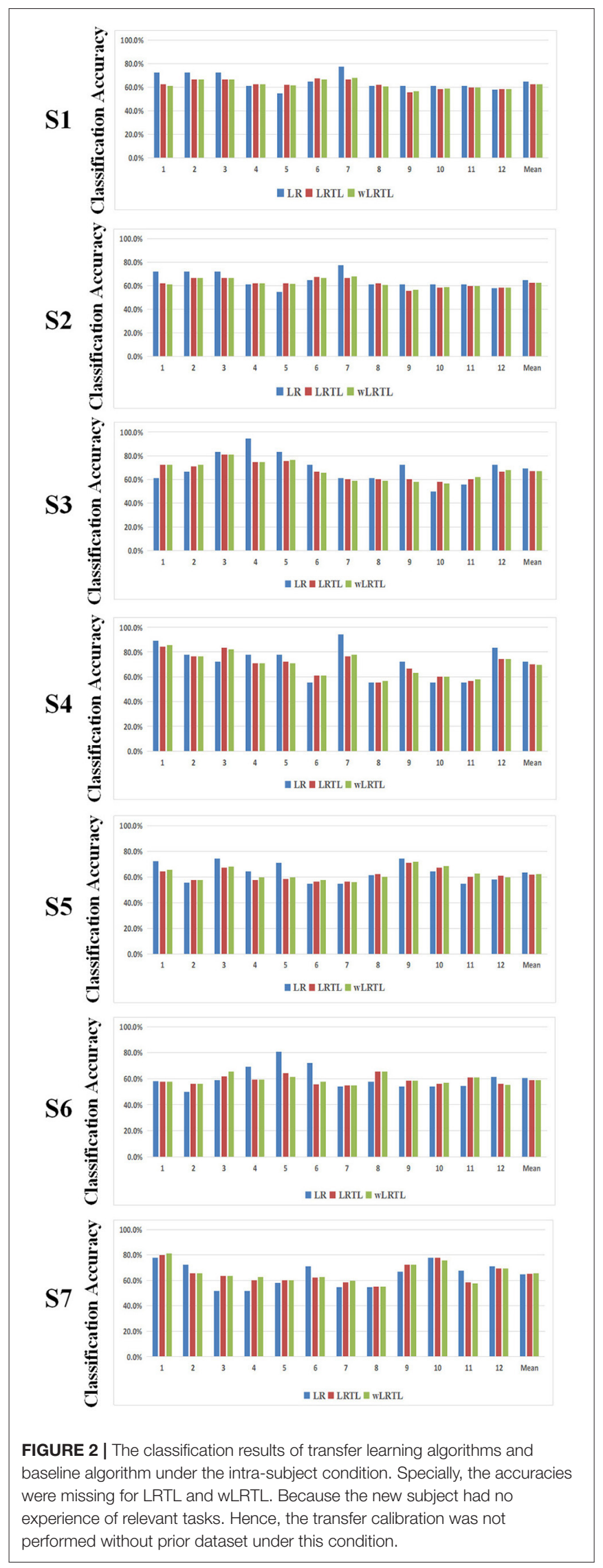

TABLE 2 | The average accuracy of low-precision sessions ( $\leq 60 \%)$ for transfer learning algorithms (i.e., LRTL, wLRTL) and baseline algorithm (i.e., LR) under the intra-subject condition.

\begin{tabular}{lccccccc}
\hline Methods & S1 & S2 & S3 & S4 & S5 & S6 & S7 \\
\hline LR (\%) & 56.5 & 53.6 & 52.8 & 55.6 & 55.6 & 54.7 & 54.2 \\
LRTL (\%) & 60.2 & 56.8 & 56.7 & 58.9 & 58.2 & 59.8 & 59.1 \\
WLRTL (\%) & 59.9 & 57.2 & 56.7 & 59.4 & 58.6 & 60.4 & 59.7
\end{tabular}

The accuracies of transfer learning approaches were higher than that of baseline approach.

TABLE 3 | The average accuracy of low-precision sessions $(\leq 60 \%)$ for transfer learning algorithms (i.e., LRTL, wLRTL) and baseline algorithm (i.e., LR) under the inter-subject condition.

\begin{tabular}{llllllll}
\hline Methods & S1 & S2 & S3 & S4 & S5 & S6 & S7 \\
\hline LR (\%) & 56.5 & 52.9 & 52.8 & 55.6 & 55.6 & 55.1 & 54.2 \\
LRTL (\%) & 60.2 & 57.3 & 58.9 & 58.3 & 58.3 & 58.9 & 59.5 \\
WLRTL (\%) & 59.9 & 57.6 & 59.4 & 58.9 & 58.7 & 59.4 & 60.2
\end{tabular}

The accuracies of transfer learning approaches were higher than that of baseline approach.

where det and $\mathrm{K}$ denoted the determinant function and the dimension of the data, respectively. Thus, the $\Sigma_{t}$ was updated as below,

$$
\Sigma_{t_{w}}=\frac{\operatorname{diag}\left(\Sigma_{t^{\prime}=1}^{n}\left(\alpha_{t^{\prime}} w_{t^{\prime}}-\mu_{t}\right)\left(\alpha_{t^{\prime}} w_{t^{\prime}}-\mu_{t}\right)^{T}\right)}{\operatorname{trace}\left(\Sigma_{t^{\prime}=1}^{n}\left(\alpha_{t^{\prime}} w_{t^{\prime}}-\mu_{t}\right)\left(\alpha_{t^{\prime}} w_{t^{\prime}}-\mu_{t}\right)^{T}\right)}
$$

where $\alpha$ and $\mu$ was computed as

$$
\begin{gathered}
\alpha_{t^{\prime}}=\frac{\left(1 /\left(K L\left[E_{s}, E_{t^{\prime}}\right]+\varepsilon\right)^{4}\right)}{\left(1 /\left(K L\left[E_{s}, E_{i}\right]+\varepsilon\right)^{4}\right)}, \\
\mu_{t}=\frac{1}{n} \sum_{t=1}^{n} \alpha_{t^{\prime}} w_{t} .
\end{gathered}
$$

Here, the divergence was calculated by averaging the $\mathrm{KL}$ divergences calculated for each class separately. The details of weighted LRTL (wLRTL) approach and the above other algorithms can be reviewed in Azab et al. (2019).

In this study, we discussed the transfer scheme for several subjective conditions of prior knowledge. Inter- and Intra-subject transfer learning approaches were both used to evaluate the methodological effectiveness. Inter-subject transfer calibration trained this classifier with prior experimental trials from other subjects while intra-subject transfer calibration performed this work using its own existing dataset. The target of our research was to find which kind of transfer strategies could be made to improve the online single-trial accuracy of BCI rehabilitation. The right bio-feedback (e.g., robotic arm) was able to raise the subjective confidence and patience to improve the therapeutic effect.

In this experiment, the performances of first sessions were unavailable for intra-subject transfer learning algorithms on 
account of no prior dataset. We used the sequential collecting of source data for transfer calibration. That meant that the target session (e.g., Session 5) would be trained by all prior collections (e.g., Session 1, Session 2, Session 3, Session 4). It was consistent with the real condition of model training. Meanwhile, the collection of source data was picked from all other subjects under the inter-subject condition. Specifically, the dataset of each subject which obtained the best performance in all sessions was used for transfer learning. Moreover, 5-fold cross validation was conducted for each approach. All 31 channels of the EEG data were selected for pattern classification. The 45 trials of MA and IS tasks (45 trials per class) were randomly divided into five sets. Four sets were used to train the classifier and the other set was tested to evaluate the performance.

\section{RESULTS}

\subsection{The Experimental Performance of Intra-Subject Based Transfer Learning Approach}

In our study, the precision of pattern recognition was considered as the most important index for BCI rehabilitation. Figure 2 lists the classification results of the above intra-subject transfer learning approaches (LRTL, wLRTL), as well as the baseline approach (LR). The average classification accuracies of all patients were higher than $60 \%$ for three different algorithms, except for P6. And it was indicated that the pattern of motor attempt could be distinctive from that of an idle state without motor attempt. However, a paired $t$-test with Bonferroni correction showed that no discriminatory differences were presented between transfer learning approaches and the baseline approach (LR vs. LRTL: $p=0.0277$; LR vs. wLRTL: $p=0.0613$; LRTL vs. wLRTL: $p=0.6085)$. This result suggests that transfer calibration did not significantly improve the BCI performance.

Furthermore, we analyzed the performance of low-precision $(\leq 60 \%)$ sessions for all patients (Table 2). Paired $t$-test with Bonferroni correction showed that the classification results of transfer learning approaches were significantly greater than that of the baseline approach (LR vs. LRTL: $p=0.0001$; LR vs. wLRTL: $p=0.0001$; LRTL vs. wLRTL: $p=0.0563$ ). It was meaningfully revealed that transfer calibration could improve the BCI performance induced by poor model training.

\subsection{Inter-Subject Based Transfer Learning Approach for MI-Based BCI Rehabilitation}

Similarly, Table 3 lists the classification results of all three algorithms under the inter-subject condition. Paired $t$-test with Bonferroni correction showed that no discriminative differences were presented between transfer learning approaches and the baseline approach (LR vs. LRTL: $p=0.0488$; LR vs. wLRTL: $p=0.1207$; LRTL vs. wLRTL: $p=0.1744$ ). This result suggested that the non-significance was consistent with those under the intra-subject condition.

Additionally, low-precision sessions were extracted for further analysis (Figure 3). In this case, the statistical analysis indicated

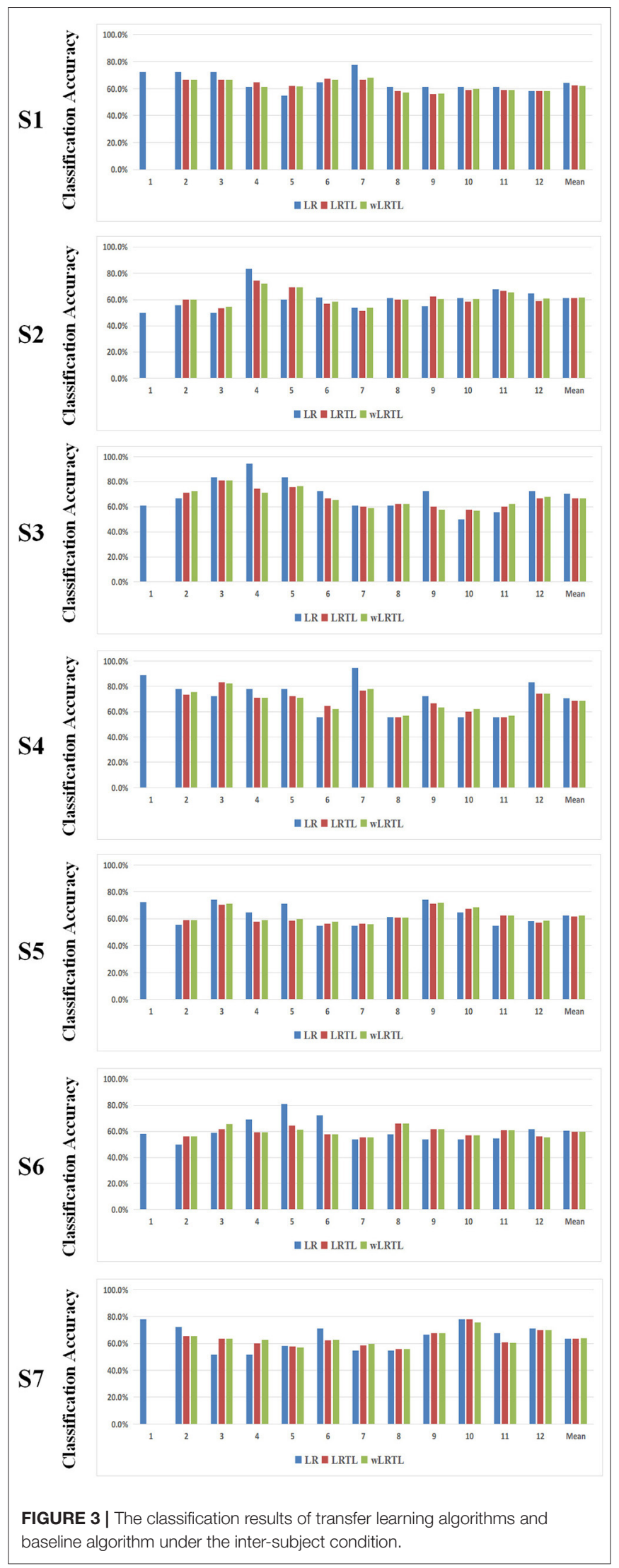




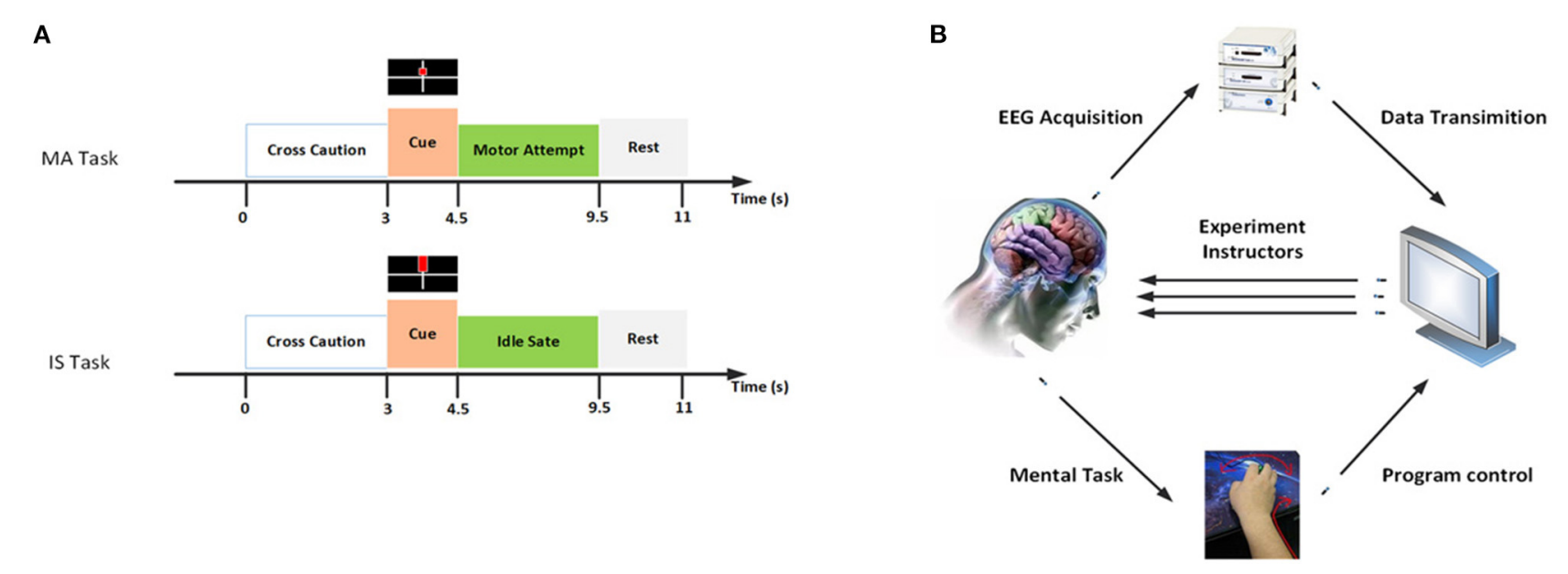

FIGURE 4 | (A) The comparison of low-precision classification results for LRTL algorithm between intra-subject and inter-subject conditions. (B) The comparison of low-precision classification results for wLRTL algorithm between intra-subject and inter-subject conditions.

that the accuracies of transfer learning approaches were significantly higher than that of the baseline approach (LR vs. LRTL: $p=0.0001$; LR vs. wLRTL: $p=0.0001$; LRTL vs. wLRTL: $p=0.0210$ ). It was revealed that transfer calibration improved the BCI performance under the inter-subject condition.

\section{DISCUSSION}

This study proposed a novel transfer calibration scheme to improve low-precision performance for BCI rehabilitation. This scheme of transfer learning could be used for new subjects without the training dataset, as well as replacing the poor training model-whose accuracy was close to the chance level. Cerebral activities were also observed to clarify the benefit of transfer calibration for feature selection.

\subsection{Improvement of Low-Precision Performance for BCI Rehabilitation}

As we know, the critical issue of BCI rehabilitation revolves around how to promote the biofeedback effect for active intervention (Ko et al., 2019). It was positive for rehabilitation outcomes, enhancing cortical activity for neural recovery, and increasing confidences of voluntary training (Zhang et al., 2020). Hence, the biofeedback of low-precision trials was negative for patients. Furthermore, most of the sessions used by the baseline classifier achieved the effective performance ( $>60 \%$ ) for each patient, except for S6. It was indicated that very few non-effective results of experimental sessions were unreliable for evaluating this neural treatment. Improved performance of transfer calibration could eliminate the confusion caused by the precision fluctuation.

Moreover, we presented the comparison of transfer learning algorithms between inter-subject (IERS) and intra-subject (IRAS) conditions (Figure 4). Statistical analysis was used to compare the classification accuracies among transfer conditions (LRTL: IERS vs. IRAS: $p=0.3138$; wLRTL: IERS vs. IRAS: $p=$
0.3501;). This result suggested that both of them were reliable for improving low-precision performance of SMR-BCI tasks. However, the advantage of inter-subject transfer calibration was employed for new subjects without training.

The fluctuation of classification for LR resulted from the differences of brain activity between consecutive sessions. It was deduced that changes of cerebral activities were caused by neural self-rehabilitation for patients. As a result, BCI-based intervention was effective for stroke rehabilitation to some extent. Nevertheless, the methodology needs to be further clarified for high-efficiency treatment.

The efficiency of transfer learning has been verified by motor imagery -based BCI tasks for healthy people. However, cerebral impairment of stroke patients would influence the training effect due to the weak neural activities. Therefore, our classification result was lower than those of the above state-of-art BCI systems (Azab et al., 2019, 2020; He and Wu, 2019). Nevertheless, the improvement of low-precision performance was conductive to treatment for the impatient. Compared to the feedback of the random level, the subject was subjectively motivated by positive feedback of right detection. It is meaningful for long-term continuous rehabilitation.

\subsection{Transfer Calibration Scheme for SMR-Based BCI Intervention}

In our study, an available scheme of transfer calibration was proposed for model selection in the online task of SMR-BCI rehabilitation (Figure 5). We summarized several rules as stated below: (1) Instant self-training was necessary for new subjects. The classification model based on current dataset was reliable for BCI tasks. (2) If the patient was frustrated by tedious training, intra- or inter-subject transfer calibration could be used to reduce the calibration time. (3) Furthermore, we could train another model when sufficient trials were finished in the task. If the precision of current model was superior to prior transfer model, the alternative could be automatically performed by our control system. (4) If the model based on the current training dataset 


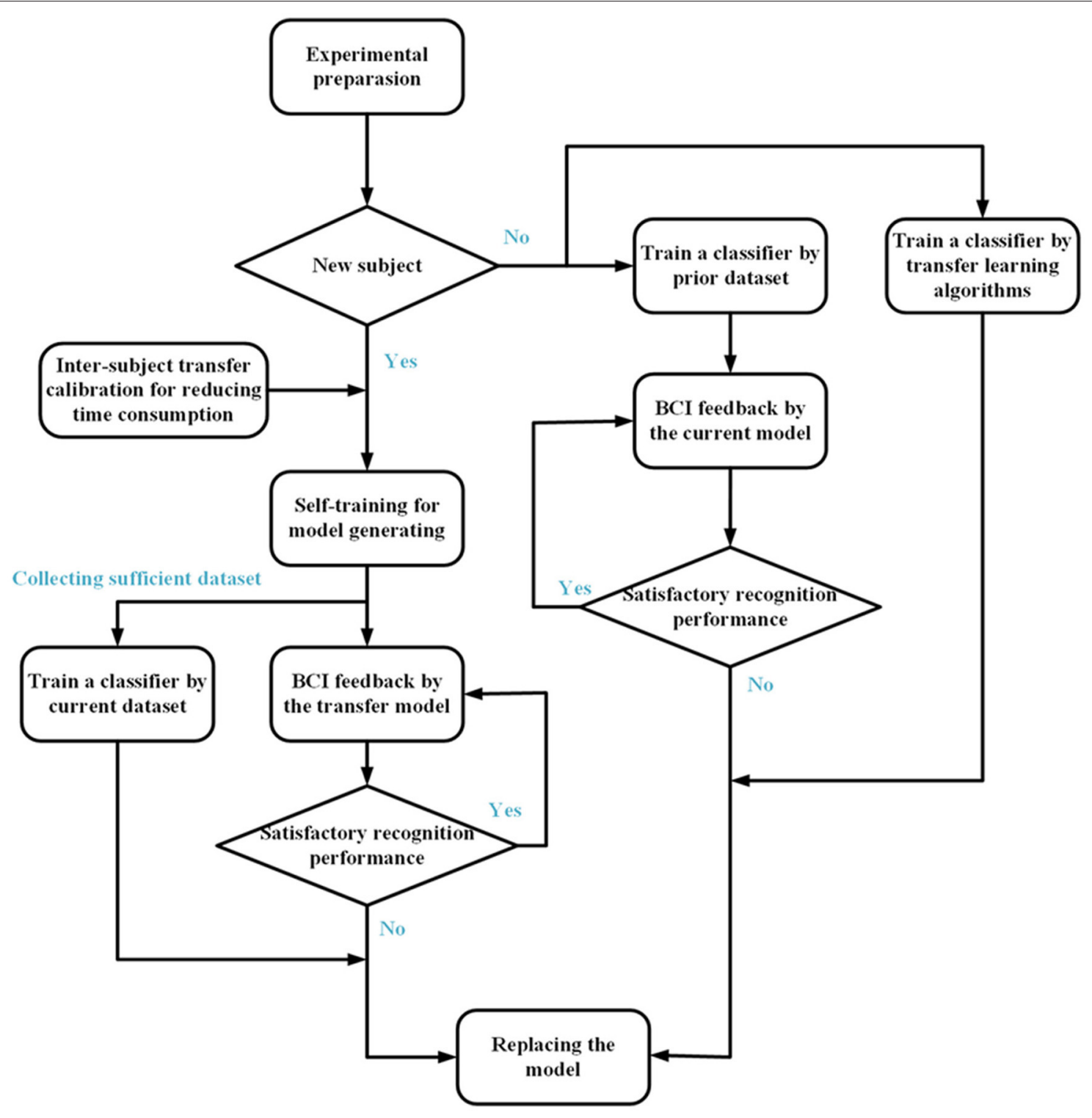

FIGURE 5 | The transfer calibration scheme of classifier model selection for SMR-BCl rehabilitation. For a new subject, intra- or inter-subject transfer calibration could be used for reducing the calibration time. Meanwhile, we could train another model when sufficient trials were finished in the task. If the precision of current using model was superior to that of the spare model, the alternative could be automatically performed by control system.

performed poorly on the experiment, intra- or inter-subject transfer calibration was worth trying in order to replace the under-performing model. (5) For transfer calibration, the volume of EEG data was a crucial factor for model selection between intra-subject and inter-subject conditions. Sufficient training data was an essential precondition for transfer calibration. Specifically, the only option for new subjects without prior experience was inter-subject transfer calibration.

This scheme of transfer calibration was feasible for improving the poor performance of SMR-BCI recognition. And it also reduced the time consumption of model calibration. It was inferred that this scheme was suitable for other transfer learning algorithms.

\subsection{Limitation of Current Work}

In this study, some issues should be noted and considered in our future work. First, the scheme of transfer calibration needed to be verified for a large amount of stroke patients. It will be addressed in future studies. Second, these patients performed these experiments for 3 months. BCI training over longer time periods should be observed to evaluate performances of the patients in different stages of post-stroke time. Moreover, the number of electrodes was supposed to be reduced by data analysis. It could reduce the time consumption of BCI rehabilitation. Thus, future studies should be conducted to solve these problems to improve the performance of online SMR-BCI rehabilitation. 


\section{CONCLUSIONS}

This paper proposed an effective scheme of transfer calibration for SMR-BCI rehabilitation. The inter- and intra-subject transfer learning approaches could improve the low-precision classification model for BCI feedback. The results imply that this systematical scheme is positive in increasing confidence of voluntary training for stroke patients. It also reduced the time consumption of model calibration.

\section{DATA AVAILABILITY STATEMENT}

The raw data supporting the conclusions of this article will be made available by the authors, without undue reservation.

\section{ETHICS STATEMENT}

The studies involving human participants were reviewed and approved by Ethics Committee of Huashan Hospital. The

\section{REFERENCES}

Arvaneh, M., Guan, C., Ang, K. K., and Quek, C. (2013). Optimizing spatial filters by minimizing within-class dissimilarities in electroencephalogrambased brain-computer interface. IEEE Trans. Neural Netw. Learn. Syst. 24, 610-619. doi: 10.1109/TNNLS.2013.2239310

Azab, A. M., Ahmadi, H., Mihaylova, L., and Arvaneh, M. (2020). Dynamic time warping-based transfer learning for improving common spatial patterns in brain-computer interface. J. Neural Eng. 17:016061. doi: 10.1088/1741-2552/ab64a0

Azab, A. M., Mihaylova, L., Ang, K. K., and Arvaneh, M. (2019). Weighted transfer learning for improving motor imagery-based brain-computer interface. IEEE Trans. Neural Syst. Rehabil. Eng. 27, 1352-1359. doi: 10.1109/TNSRE.2019.2923315

Azab, A. M., Toth, J., Mihaylova, L. S., and Arvaneh, M. (2018). “A review on transfer learning approaches in brain-computer interface," in Signal Processing and Machine Learning for Brain-Machine Interfaces, eds T. Tanaka, and M. Arvaneh (Sheffield: IET), 81-98. doi: 10.1049/PBCE114E_ch5

Birbaumer, N. (2006). Breaking the silence: brain-computer interfaces (BCI) for communication and motor control. Psychophysiology 43, 517-532. doi: 10.1111/j.1469-8986.2006.00456.x

Buch, E., Weber, C., Cohen, L. G., Braun, C., Dimyan, M. A., Ard, T., et al. (2008). Think to move: a neuromagnetic brain-computer interface (BCI) system for chronic stroke. Stroke 39, 910-917. doi: 10.1161/STROKEAHA.107.505313

Bundy, D. T., Souders, L., Baranyai, K., Leonard, L., Schalk, G., Coker, R., et al. (2017). Contralesional brain-computer interface control of a powered exoskeleton for motor recovery in chronic stroke survivors. Stroke 48, 1908-1915. doi: 10.1161/STROKEAHA.116.016304

Caria, A., da Rocha, J. L. D., Gallitto, G., Birbaumer, N., Sitaram, R., and Murguialday, A. R. (2020). Brain-machine interface induced morphofunctional remodeling of the neural motor system in severe chronic stroke. Neurotherapeutics 17, 635-650. doi: 10.1007/s13311-019-00816-2

De Vries, S., Tepper, M., Feenstra, W., Oosterveld, H., Boonstra, A. M., and Otten, B. (2013). Motor imagery ability in stroke patients: the relationship between implicit and explicit motor imagery measures. Front. Hum. Neurosci. 7:790. doi: 10.3389/fnhum.2013.00790

Foong, R., Ang, K. K., Quek, C., Guan, C., Phua, K. S., Kuah, C. W. K., et al. (2019). Assessment of the efficacy of EEG-based MI-BCI with visual feedback and EEG correlates of mental fatigue for upper-limb stroke rehabilitation. IEEE Trans. Biomed. Eng. 67, 786-795. doi: 10.1109/TBME.2019.2921198

Gao, S., Zhou, M., Wang, Y., Cheng, J., Yachi, H., and Wang, J. (2019). Dendritic neural model with effective learning algorithms for classification, patients/participants provided their written informed consent to participate in this study.

\section{AUTHOR CONTRIBUTIONS}

LC and HW designed the methodology of data process and performed the data analysis. LC and SC organized the data and wrote the manuscript. LC, ZX, CF, and JJ reviewed and edited the manuscript. All authors read and approved the submitted manuscript.

\section{FUNDING}

This study was supported by the National Natural Science Foundation of China (Grant No. 61806122), Project of Dai Kerong Academician Workstation (Grant No. MS201758), and Wuxi Health Commission Research Project of China (Grant Nos. ME201944 and T201906).

approximation, and prediction. IEEE Trans. Neural Netw. Learn. Syst. 30, 601-604. doi: 10.1109/TNNLS.2018.2846646

Grosse-Wentrup, M., Mattia, D., and Oweiss, K. (2011). Using brain-computer interfaces to induce neural plasticity and restore function. J. Neural Eng. 8:025004. doi: 10.1088/1741-2560/8/2/025004

$\mathrm{He}, \mathrm{H}$., and $\mathrm{Wu}, \mathrm{D}$. (2019). Transfer learning for brain-computer interfaces: a Euclidean space data alignment approach. IEEE Trans. Biomed. Eng. 67, 399-410. doi: 10.1109/TBME.2019.2913914

Hossain, I., Khosravi, A., Hettiarachchi, I., and Nahavandi, S. (2018). Multiclass informative instance transfer learning framework for motor imagerybased brain-computer interface. Comput. Intell. Neurosci. 2018, 1-12. doi: 10.1155/2018/6323414

Huang, D., Qian, K., Fei, D.-Y., Jia, W., Chen, X., and Bai, O. (2012). Electroencephalography (EEG)-based brain-computer interface (BCI): a 2-D virtual wheelchair control based on event-related desynchronization/synchronization and state control. IEEE Trans. Neural Syst. Rehabil. Eng. 20, 379-388. doi: 10.1109/TNSRE.2012.2 190299

Jayaram, V., Alamgir, M., Altun, Y., Scholkopf, B., and Grosse-Wentrup, M. (2016). Transfer learning in brain-computer interfaces. IEEE Comput. Intell. Mag. 11, 20-31. doi: 10.1109/MCI.2015.2501545

Kang, H., Nam, Y., and Choi, S. (2009). Composite common spatial pattern for subject-to-subject transfer. IEEE Signal Process. Lett. 16, 683-686. doi: 10.1109/LSP.2009.2022557

Ko, L.-W., Lu, Y.-C., Bustince, H., Chang, Y.-C., Chang, Y., Ferandez, J., et al. (2019). Multimodal fuzzy fusion for enhancing the motor-imagerybased brain computer interface. IEEE Comput. Intell. Mag. 14, 96-106. doi: 10.1109/MCI.2018.2881647

Kobler, R. J., and Scherer, R. (2016). "Restricted boltzmann machines in sensory motor rhythm brain-computer interfacing: a study on inter-subject transfer and co-adaptation," in 2016 IEEE International Conference on Systems, Man, and Cybernetics (SMC) (Budapest: IEEE), 469-474. doi: 10.1109/SMC.2016. 7844284

Mane, R., Chew, E., Phua, K. S., Ang, K. K., Robinson, N., Vinod, A., et al. (2019). Prognostic and monitory EEG-biomarkers for BCI upper-limb stroke rehabilitation. IEEE Trans. Neural Syst. Rehabil. Eng. 27, 1654-1664. doi: 10.1109/TNSRE.2019.2924742

Mane, R., Chouhan, T., and Guan, C. (2020). Bci for stroke rehabilitation: motor and beyond. J. Neural Eng. 17:041001. doi: 10.1088/1741-2552/aba162

Meng, J., Zhang, S., Bekyo, A., Olsoe, J., Baxter, B., and He, B. (2016). Noninvasive electroencephalogram based control of a robotic arm for reach and grasp tasks. Sci. Rep. 6:38565. doi: 10.1038/srep38565 
Pfurtscheller, G., and Da Silva, F. L. (1999). Event-related EEG/MEG synchronization and desynchronization: basic principles. Clin. Neurophysiol. 110, 1842-1857. doi: 10.1016/S1388-2457(99)00141-8

Pichiorri, F., Morone, G., Petti, M., Toppi, J., Pisotta, I., Molinari, M., et al. (2015). Brain-computer interface boosts motor imagery practice during stroke recovery. Ann. Neurol. 77, 851-865. doi: 10.1002/ana. 24390

Pillette, L., Lotte, F., N'Kaoua, B., Joseph, P.-A., Jeunet, C., and Glize, B. (2020). Why we should systematically assess, control and report somatosensory impairments in BCI-based motor rehabilitation after stroke studies. Neuroimage Clin. 28:102417. doi: 10.1016/j.nicl.2020. 102417

Ramos-Murguialday, A., Broetz, D., Rea, M., Läer, L., Yilmaz, Ö., Brasil, F. L., et al. (2013). Brain-machine interface in chronic stroke rehabilitation: a controlled study. Ann. Neurol. 74, 100-108. doi: 10.1002/ana.23879

Remsik, A. B., Williams, L. Jr., Gjini, K., Dodd, K., Thoma, J., Jacobson, T., et al. (2019). Ipsilesional Mu rhythm desynchronization and changes in motor behavior following post stroke BCI intervention for motor rehabilitation. Front. Neurosci. 13:53. doi: 10.3389/fnins.2019.00053

Saha, S., Ahmed, K. I. U., Mostafa, R., Hadjileontiadis, L., and Khandoker, A. (2017). Evidence of variabilities in EEG dynamics during motor imagery-based multiclass brain-computer interface. IEEE Trans. Neural Syst. Rehabil. Eng. 26, 371-382. doi: 10.1109/TNSRE.2017.2778178

Samek, W., Kawanabe, M., and Müller, K.-R. (2013a). Divergence-based framework for common spatial patterns algorithms. IEEE Rev. Biomed. Eng. 7, 50-72. doi: 10.1109/RBME.2013.2290621

Samek, W., Meinecke, F. C., and Müller, K.-R. (2013b). Transferring subspaces between subjects in brain-computer interfacing. IEEE Trans. Biomed. Eng. 60, 2289-2298. doi: 10.1109/TBME.2013.2253608

Shu, X., Chen, S., Meng, J., Yao, L., Sheng, X., Jia, J., et al. (2018). Tactile stimulation improves sensorimotor rhythm-based BCI performance in stroke patients. IEEE Trans. Biomed. Eng. 66, 1987-1995. doi: 10.1109/TBME.2018.2882075
Vidaurre, C., Kawanabe, M., von Bünau, P., Blankertz, B., and Müller, K.-R. (2010). Toward unsupervised adaptation of lda for brain-computer interfaces. IEEE Trans. Biomed. Eng. 58, 587-597. doi: 10.1109/TBME.2010.2093133

Wang, Y., Gao, S., and Gao, X. (2006). "Common spatial pattern method for channel selelction in motor imagery based brain-computer interface," in 2005 IEEE Engineering in Medicine and Biology 27th Annual Conference (Shanghai: IEEE), 5392-5395. doi: 10.1109/IEMBS.2005.1615701

$\mathrm{Wu}$, D. (2016). Online and offline domain adaptation for reducing BCI calibration effort. IEEE Trans. Hum. Mach. Syst. 47, 550-563. doi: 10.1109/THMS.2016.2608931

Xu, R., Jiang, N., Vuckovic, A., Hasan, M., Mrachacz-Kersting, N., Allan, D., et al. (2014). Movement-related cortical potentials in paraplegic patients: abnormal patterns and considerations for BCI-rehabilitation. Front. Neuroeng. 7:35. doi: 10.3389/fneng.2014.00035

Zhang, K., Xu, G., Zheng, X., Li, H., Zhang, S., Yu, Y., et al. (2020). Application of transfer learning in EEG decoding based on brain-computer interfaces: a review. Sensors 20:6321. doi: 10.3390/s20216321

Zhao, X., Zhao, J., Cai, W., and Wu, S. (2019). Transferring common spatial filters with semi-supervised learning for zero-training motor imagery brain-computer interface. IEEE Access 7, 58120-58130. doi: 10.1109/ACCESS.2019.2913154

Conflict of Interest: The authors declare that the research was conducted in the absence of any commercial or financial relationships that could be construed as a potential conflict of interest.

Copyright (C) 2021 Cao, Chen, Jia, Fan, Wang and Xu. This is an open-access article distributed under the terms of the Creative Commons Attribution License (CC BY). The use, distribution or reproduction in other forums is permitted, provided the original author(s) and the copyright owner(s) are credited and that the original publication in this journal is cited, in accordance with accepted academic practice. No use, distribution or reproduction is permitted which does not comply with these terms. 